\title{
Relic Gravitons and String Pre-big-bang Cosmology
}

\author{
A. Buonanno ${ }^{1}$ and C. Ungarelli ${ }^{2}$ \\ 1 Physics Department, University of Maryland, College Park, MD 20742, USA \\ buonanno@umd.edu \\ 2 Physics Department "Enrico Fermi", University of Pisa, Largo Pontecorvo 3, \\ 56127 Pisa, Italy and Geosciences and Earth Resources Institute, CNR, \\ via Moruzzi 1, 56124 Pisa, Italy \\ carlo.ungarelli@igg.cnr.it
}

\begin{abstract}
In this paper, after discussing the mechanism of graviton production during an early phase of accelerated expansion, we will review the main features of the spectrum of primordial gravitational radiation for the class of string-inspired models called pre-big-bang models. Furthermore, we will also outline the implications on pre-big-bang models of current and future searches of gravitational waves with ground-based detectors.
\end{abstract}

\section{Foreword}

This contribution reviews one of the many research topics originally pioneered by Gabriele Veneziano in String theory and fundamental interactions. We are thankful to Gabriele for having taught us not only physics, but also how to be good physicists, choosing and tackling problems with deepness and seriousness. He will continue to be for us a precious source of inspiration.

\section{Introduction}

In recent years, a number of detectors have been designed and built to search for gravitational waves (GWs). Ground-based interferometers aimed at detecting GWs in the frequency range between $10 \mathrm{~Hz}$ and $1 \mathrm{kHz}$, such as LIGO [1, VIRGO [2], GEO600 [3], and TAMA 4] are now operating at design sensitivity (or close to it in the case of VIRGO). The design of a space-based three-arm interferometer, the Laser Interferometer Space Antenna (LISA) [5], will explore the frequency window between 0.1 and $10 \mathrm{mHz}$. A second generation of space-based detector probing primordial GWs [6, 7] is under planning. Following earlier theoretical works [8], prototypes for detecting high-frequency 
GWs, in the millihertz band, have been developed [9]. Finally, the large number of millisecond pulsar detectable with the square kilometer array (SKA) 10] would provide an ensemble of clocks that can be used as multiple arms of a GW detector in the frequency range around $10^{-9} \mathrm{~Hz}$.

One of the possible targets of such search is a stochastic gravitational-wave background (SGWB). Depending on its origin, the stochastic background can be broadly divided into two classes (for a review see, e.g., [1, 12]): the astrophysically generated background due to the incoherent superposition of gravitational radiation emitted by large populations of astrophysical sources that cannot be resolved individually, and the primordial GW background generated by processes taking place in the early stages of the Universe. A primordial component of such background is especially interesting, since it would carry unique information about the state of the primordial Universe. Here we focus our attention on a particular type of primordial stochastic background, namely the relic radiation produced by the parametric amplification of metric tensor perturbations during an early stage of accelerated expansion (inflationary stage) [13. Leaving the detailed analysis of the production mechanism to the following section, the energy and spectral content of such radiation is encoded in the spectrum, defined as follows:

$$
\Omega_{\mathrm{GW}}=\frac{1}{\rho_{c}} f \tilde{\rho}_{\mathrm{GW}}(f),
$$

where $f$ is the frequency, $\rho_{c}$ is the critical energy density of the Universe $\left(\rho_{c}=3 H_{0}^{2} / 8 \pi G\right)$ and $\tilde{\rho}_{\mathrm{GW}}$ is the GWs energy density per unit frequency, i.e.,

$$
\rho_{\mathrm{GW}}=\int_{0}^{\infty} d f \tilde{\rho}_{\mathrm{GW}}(f) .
$$

For a spectrum produced during an early stage of slow-roll inflation, the spectrum decreases as $f^{-2}$ in the frequency window $10^{-18}-10^{-16} \mathrm{~Hz}$, and then slowly decreases up to a frequency corresponding to modes whose physical frequency becomes less than the maximum causal distance during the reheating phase (which is of order of a few gigahertz). For this class of models, the spectral content of the SGWB is fixed in terms of the shape parameters of the inflaton potential. Its magnitude depends on both the value of the Hubble parameter during inflation and a number of features characterizing the Universe evolution after the inflationary era - for example, tensor anisotropic stress due to free-streaming relativistic particles, equations of state [14, 15, and so on. An upper bound on the spectrum can be obtained from the measurement of the quadrupole anisotropy of the cosmic microwave background (CMB). Through the Sachs-Wolfe effect, a SGWB at large scales (i.e., at wavelengths comparable to the present value of the Hubble radius) would induce stochastic anisotropies in the CMB temperature. This yields an upper limit of $h_{0}^{2} \Omega_{\mathrm{GW}} \sim 5 \times 10^{-15}$ at $f \sim 10^{-16} \mathrm{~Hz}$ [16. Since for a generic slow-roll inflationary model the spectrum is (weakly) decreasing with frequency (for a recent 
review see, e.g., [15, 17]), this implies an upper bound $h_{0}^{2} \Omega_{\mathrm{GW}} \sim 5 \times 10^{-16}$ at frequencies around $f \sim 100 \mathrm{~Hz}$, where ground-based detectors such as LIGO reach the best sensitivity. For a flat spectrum, the recent LIGO results [18] sets an upper limit $h_{0}^{2} \Omega_{\mathrm{GW}}<6.5 \times 10^{-5}$. For frequency-independent spectra, the expected upper limit for the current LIGO configuration is $h_{0}^{2} \Omega_{\mathrm{GW}}<5 \times 10^{-6}$, while the advanced LIGO project design sensitivity is $h_{0}^{2} \Omega_{\mathrm{GW}} \sim 8 \times 10^{-9}$ (see, e.g., [19]).

The spectrum predicted by the class of single-field inflationary models is then too low to be observed by ground-based detectors. It is therefore evident that a background satisfying the bound imposed by the observed amount of CMB anisotropies at large scales could be detected at frequencies relevant for ground-based GW detectors provided that its spectrum grows significantly with frequency.

Pre-big-bang (PBB) models, originally proposed by Veneziano [20], and then Gasperini and Veneziano 21] (for a detailed review, see [22]), represent an interesting class of inflationary models alternative to the standard slowroll ones. In particular, the presence in the inflationary phase of fields like the dilaton or moduli, can have important consequences on the spectral properties of the SGWB, thus affecting the possibility of detection by earth-based interferometers. As first shown in 23 , at low frequencies, say $f \gtrsim 10^{-16} \mathrm{~Hz}$, the SGWB spectrum grows as $\Omega_{\mathrm{GW}} \sim f^{3}$. Hence, the COBE bound is easily evaded and the spectrum can peak at frequencies around $10-10^{3} \mathrm{~Hz}$, still satisfying the bound from big-bang nucleosynthesis (BBN) 24] and CMB [25].

The aim of this paper is both to review the general mechanism of cosmological graviton production, describing its key features, and discuss the prospect of detection within the class of PBB cosmological models. The paper is organized as follows. In Sect. 2 we review the mechanism of parametric amplification of metric perturbations, and discuss examples in De Sitter and slow-roll inflation. In Sect. 3 we compute the SGWB in non-minimal PBB models and discuss the main modifications in non-minimal models. In Sect. 4 we review the implications of current and future results of ground-based detector (in particular the LIGOs) on PBB models. Finally, in Sect. 5 we draw some conclusions.

\section{Graviton Production in Cosmology}

One of the most relevant aspects of inflationary models is that they provide a natural mechanism for generating perturbations in all matter fields. Such primordial perturbations can then be considered as seeds for the observed CMB anisotropies and large-scale structures, and can also yield to a SGWB. Those observable consequences are all related to the well-known phenomenon of amplification of quantum-vacuum fluctuations in cosmological backgrounds [13]. In this section, starting with the simple toy model of a 
one-dimensional harmonic oscillator [26], we shall compute the SGWB in De Sitter inflation and slow-roll inflation.

Let us consider a one-dimensional harmonic oscillator moving in an expanding background described by a scale factor $a(t)$. The Lagrangian is

$$
L=\frac{a^{2} m}{2}\left(\dot{x}^{2}-\omega^{2} x^{2}\right)
$$

the canonical momentum and the corresponding Hamiltonian-computed as the Legendre transformation of the Lagrangian (3) - read

$$
\begin{gathered}
p=a^{2} m \dot{x} \\
H=\frac{1}{2}\left(\frac{p^{2}}{a^{2} m}+a^{2} m \omega^{2} x^{2}\right) .
\end{gathered}
$$

The corresponding equations of motion are

$$
\begin{gathered}
\ddot{x}+2 \frac{\dot{a}}{a} \dot{x}+\omega^{2} x=0, \\
\ddot{y}+\left(\omega^{2}-\frac{\ddot{a}}{a}\right) y=0,
\end{gathered}
$$

where we denote with a dot the derivative with respect to the cosmic time $t$ and $y=a x$ is the proper physical amplitude of the harmonic oscillator. Without specifying the details of the cosmological evolution, the properties of the solutions of (6) and (7) can be derived by analyzing their behavior in two different regimes:

(a) When $\omega^{2} \gg \ddot{a} / a$, the comoving amplitude and momentum are adiabatically damped

$$
x \sim \frac{1}{a} e^{i \omega t}, \quad p \sim a \omega e^{i \omega t} .
$$

Hence, in this regime the proper physical amplitude and momentum are approximately constant (as well as the Hamiltonian (5));

(b) For $\omega^{2} \ll \ddot{a} / a$ the comoving amplitude and momentum are frozen

$$
x \sim B+C \int_{0}^{t} d t^{\prime} \frac{1}{a^{2}\left(t^{\prime}\right)}, \quad p \sim C .
$$

Notice that in this freeze-out regime

$$
\frac{d}{d t}\left(\frac{\lambda_{\text {phys }}}{H^{-1}}\right)>0
$$

where $\lambda_{\text {phys }}=2 \pi a / \omega$ is the proper physical wavelength of the oscillator and $H=\dot{a} / a$ is the expansion rate. The condition (10) implies that the background expansion is accelerating (as it occurs during an inflationary phase) 
and the proper wavelength characteristic of the oscillator is expanding faster than the maximum causal distance $H^{-1}$. Furthermore, during such freeze-out regime the value of the energy is asymptotically dominated by the term proportional to $x^{2}$ and is due to the stretching of the oscillator produced by the rapidly accelerated expansion. Let us now consider a cosmological evolution characterized by the following three different phases:

$$
\begin{gathered}
\omega^{2}>\ddot{a} / a \quad t<t_{\mathrm{ex}}, t>t_{\mathrm{re}}, \\
\omega^{2}<\ddot{a} / a \quad t_{\mathrm{ex}}<t<t_{\mathrm{re}} .
\end{gathered}
$$

By smoothly joining the solution of the equations of motion (6) and (7) in the three different phases, it is straightforward to show that the final energy $E_{\text {fin }}$ of the harmonic oscillator (which is asymptotically constant during the initial and final phases) is enhanced during the intermediate, accelerating phase by a factor proportional to $\left[a\left(t_{\mathrm{re}}\right) / a\left(t_{\mathrm{ex}}\right)\right]^{2}$

$$
E_{f} \sim\left[\frac{a\left(t_{\mathrm{re}}\right)}{a\left(t_{\mathrm{ex}}\right)}\right]^{2} E_{i n} .
$$

Note that for a classical oscillator initially at rest $\left(x_{\text {in }}=p_{\text {in }}=0\right)$ the initial energy is zero and no amplification takes place. Within the same cosmological evolution described by (11) and (12), let us consider instead a one-dimensional quantum mechanical oscillator initially in the ground state. The initial wave function is

$$
\psi_{\text {in }}(x)=\left(\frac{\alpha_{\text {in }}}{\pi}\right)^{1 / 4} e^{-\alpha_{\text {in }} x^{2} / 2},
$$

where $\alpha_{\text {in }}=a^{2}\left(t_{\text {ex }}\right) m \omega^{2} / \hbar$. In the final stage of the cosmological evolution, the harmonic oscillator will be in a high occupation number state 1 This can be shown by computing the expectation value of the Hamiltonian (defined in the final stage) with respect to the initial vacuum state defined by the wave function (14). In the final stage of the cosmological evolution, the Hamiltonian operator can be approximated as

$$
\hat{H}_{f}=-\frac{\hbar^{2}}{2 m_{\mathrm{ex}}} \frac{d^{2}}{d x^{2}}+\frac{m_{\mathrm{ex}} \omega^{2} x^{2}}{2},
$$

where $m_{\mathrm{ex}}=a^{2}\left(t_{\mathrm{ex}}\right) m$. Expressing the wavefunction (14) in terms of the eigenfunctions of (15), one obtains (see, e.g., [11])

$$
\psi_{\mathrm{in}}(x)=\sum_{n=0}^{\infty} \beta_{2 n} \psi_{\mathrm{fin}}^{(n)}(x)
$$

\footnotetext{
${ }^{1}$ More precisely in a squeezed state.
} 


$$
\begin{gathered}
\beta_{2 n}=\left(\alpha_{\mathrm{in}} \alpha_{\mathrm{fin}}\right)^{1 / 4} \sqrt{\frac{2(2 n) !}{\alpha_{\mathrm{in}}+\alpha_{\mathrm{fin}}}} \frac{1}{n !}\left[\frac{\omega_{\mathrm{fin}}-\omega_{\mathrm{in}}}{2\left(\omega_{\mathrm{fin}}+\omega_{\mathrm{in}}\right)}\right]^{n}, \\
\psi_{\mathrm{fin}}^{(n)}(x)=N_{n} H_{n}\left(\sqrt{\alpha_{\mathrm{fin}}} x\right) e^{-\alpha_{\mathrm{fin}} x^{2} / 2},
\end{gathered}
$$

where $\alpha_{\text {fin }}=a\left(t_{\mathrm{re}}\right)^{2} m \omega^{2} / \hbar, \omega_{\mathrm{in}, \mathrm{fin}}=\omega / a\left(t_{\mathrm{ex}, \mathrm{re}}\right), H_{n}$ are Hermite polynomials, and $N_{n}$ is a normalization constant. Using (18) it is straightforward to show that the expectation value of the Hamiltonian (15) on the initial state described by the wavefunction (14) is given by

$$
E_{\text {fin }}=\hbar \omega_{\text {fin }}\left[\frac{1}{2}+\left(\frac{\omega_{\text {fin }}-\omega_{\text {in }}}{2 \sqrt{\omega_{\text {in }} \omega_{\text {fin }}}}\right)^{2}\right] \text {. }
$$

Hence, for a sufficiently long intermediate phase (for which $\omega_{\text {fin }} \ll \omega_{\text {in }}$ ) the harmonic oscillator final state is a semiclassical state characterized by a large number of created quanta

$$
N_{f} \sim \frac{1}{4}\left(\frac{\omega_{\text {in }}}{\omega_{\text {fin }}}\right) .
$$

This simple example shows how a period of accelerated expansion (i.e., an inflationary phase) can generate large scale inhomogeneities and anisotropies. Barring some technical issues (the conditions that guarantee the existence of a well-defined vacuum state at the onset of the inflationary phase), every quantum field in the vacuum state can be described as a collection of harmonic oscillators. The occurrence of a phase of accelerated expansion produces an amplification of the vacuum energy of those oscillators, stretching their corresponding wavelengths to scales larger than the horizon $(k<a H)$. Such modes then eventually re-enter the horizon $(k>a H)$ later, during the radiation/matter-dominated phases of the Universe evolution. Since those matter fields are gravitationally coupled (at least minimally) to the gravitational field, such enhancement of vacuum fluctuations is transferred to the background metric, therefore yielding to perturbations. Within the class of homogeneous and isotropic cosmological models, the perturbations can be classified in terms of their properties under coordinate transformations corresponding to the space-time isometries. (The latter are represented by the group $S O(3)$ of three-dimensional rotations, for a review, see [28.) In particular, scalar perturbations (described by fields invariant under rotations) are coupled to ordinary matter and radiation fields during the radiation/matter dominated phases, thus they are the seeds for large-scale structures and CMB anisotropies. Tensorial perturbations which are described by a field that transforms as a rank 2 tensor under rotations, produce a characteristic spectrum of stochastic gravitational radiation, whose energy and spectral content both depend on the background evolution and carries a unique imprint of the inflationary phase. 


\subsection{De Sitter Inflation}

For the sake of simplicity, let us consider a simplified two-stage cosmological model where the epoch of accelerated expansion is described by a De Sitter phase smoothly connected to a standard radiation dominated phase. In conformal coordinates, the space-time metric is given by

$$
d s^{2}=a(\eta)^{2}\left(d \eta^{2}-d \boldsymbol{x}^{2}\right)
$$

where $\eta$ is the conformal time. The background evolution is specified by the following expressions for the scale factor:

$$
\begin{gathered}
a(\eta)=-\frac{1}{H_{\mathrm{ds}} \eta}, \quad \eta<\eta_{1}<0 \\
a(\eta)=\frac{1}{H_{\mathrm{ds}} \eta_{1}^{2}}\left(\eta-2 \eta_{1}\right)
\end{gathered}
$$

For each comoving wave number $k$, we add transverse and traceless fluctuations of the metric described by the following tensor:

$$
h_{a b}^{(A)}(\boldsymbol{k}, \eta)=e_{a b}^{A}(\boldsymbol{k}) \tilde{h}_{k}^{(A)}(\eta) \mathrm{e}^{i \boldsymbol{k} \cdot \boldsymbol{x}},
$$

where $a, b=1,2,3, A=+, \times$ labels the polarization state described by the tensor $e_{a b}^{(A)}$ and $\boldsymbol{k}$ is the comoving wave vector. The amplitude $h_{k}^{(A)}$ satisfies the following equation:

$$
\left(\frac{d^{2}}{d \eta^{2}}+2 \mathcal{H} \frac{d}{d \eta}+|\boldsymbol{k}|^{2}\right) h_{k}^{(A)}=0,
$$

where $\mathcal{H}=(1 / a) d a / d \eta$. The general solution of (25) can be written in terms of elementary functions (in this case half-integer Hankel functions). In particular, imposing that for $\eta \rightarrow-\infty$ the solution of (25) corresponds to a vacuum state, one obtains 27.

$$
\begin{gathered}
h_{k}^{(A)}=\frac{a\left(\eta_{1}\right)}{a(\eta)}\left[1+H_{\mathrm{ds}} \omega^{-1}\right] e^{-i k\left(\eta-\eta_{1}\right)}, \quad \eta<\eta_{1}, \\
h_{k}^{(A)}=\frac{a\left(\eta_{1}\right)}{a(\eta)}\left[\alpha_{k} e^{-i k\left(\eta-\eta_{1}\right)}+\beta_{k} e^{i k\left(\eta-\eta_{1}\right)}\right], \quad \eta>\eta_{1},
\end{gathered}
$$

where $\omega=c k / a$ and $\alpha_{k}, \beta_{k}$ are the so-called Bogoliubov coefficients relative to the transition from a De Sitter to the radiation-dominated regime. In particular, for $\eta \rightarrow+\infty\left|\beta_{k}\right|^{2}$ represents the number of gravitons created per unit cell of the phase space. The Bogoliubov coefficients can be computed by imposing the continuity of the amplitude and its time derivative on the space-like surface $\eta=\eta_{1}$ [27]: 


$$
\begin{gathered}
\alpha_{k}=1+i \frac{\sqrt{H_{0} H_{\mathrm{ds}}}}{\omega}-\frac{H_{0} H_{\mathrm{ds}}}{2 \omega^{2}}, \\
\beta_{k}=\frac{H_{0} H_{\mathrm{ds}}}{2 \omega^{2}} .
\end{gathered}
$$

The graviton energy density per unit cell of phase is therefore

$$
d \rho_{\mathrm{GW}}=2 \hbar \omega\left(\frac{\omega^{2} d \omega}{2 \pi^{2}}\right)\left|\beta_{k}\right|^{2}=\frac{\hbar H_{0}^{2} H_{\mathrm{ds}}^{2}}{4 \pi^{2}} \frac{d f}{f},
$$

where $f=\omega / 2 \pi$ is the physical frequency and $H_{0}$ is the present value of the Hubble constant. Using the definition (1) the spectrum turns out to be scale-invariant and its value reads

$$
\Omega_{\mathrm{GW}}=\frac{16}{9}\left(\frac{M_{\mathrm{inf}}}{M_{\mathrm{pl}}}\right)^{4},
$$

where $M_{\mathrm{pl}}=G^{-1 / 2}=1.22 \times 10^{19} \mathrm{GeV}$ is the Planck mass and $M_{\text {inf }}$ is the inflationary scale defined by $H_{\mathrm{ds}}^{2}=8 \pi M_{\mathrm{infl}}^{4} / 3 M_{\mathrm{pl}}^{2}$. This result cannot be directly compared with experimental sensitivities, since the presence of a matter dominated and a dark energy phase is not properly taken into account. However, for modes that at the time of radiation-matter equality have physical wavelengths smaller than the horizon (corresponding to frequencies today $f>\left(H_{0} / 2 \pi\right)\left(1+z_{\text {eq }}\right)^{1 / 2}, z_{\text {eq }}$ being the redshift of matter-radiation equality), the frequency dependence is not affected by the presence of matter and dark energy-dominated eras. The corresponding spectrum is reduced by a factor $1 /\left(1+z_{\text {eq }}\right)$ with respect to $(31)$ and is given by

$$
\Omega_{\mathrm{GW}}=\frac{16}{9}\left(\frac{M_{\mathrm{infl}}}{M_{\mathrm{pl}}}\right)^{4}\left(\frac{\Omega_{r}}{1-\Omega_{\mathrm{de}}}\right),
$$

where $\Omega_{r}$ are the current fractions of radiation and dark energy densities in units of the critical energy density, respectively. Current WMAP data place an upper limit on the inflation scale around $M_{\mathrm{inf}} \sim 2 \times 10^{16} \mathrm{GeV}[29]$. Since the total energy in radiation is approximately $h_{0}^{2} \Omega_{r} \sim 4.15 \times 10^{-5}$, assuming $\Omega_{\mathrm{de}}=0.7$, one finds for the spectrum (32)

$$
h_{0}^{2} \Omega_{\mathrm{GW}}<1.7 \times 10^{-15} .
$$

\subsection{Slow-roll Inflation}

In the previous section we have focused our attention to a simplified inflationary model where the phase of accelerated expansion is driven by a constant energy density. However, a more general class of inflationary models is characterized by a scalar field $\Phi$ slowly rolling in a potential $V(\Phi)$. For such models, the expansion rate is not constant during the accelerating period and this 
feature produces a small tilt in the spectrum. In particular, for frequencies $f>\left(H_{0} / 2 \pi\right)\left(1+z_{\text {eq }}\right)^{1 / 2}$ the spectrum has a power-law frequency dependence

$$
\Omega_{\mathrm{GW}} \sim f^{n_{T}}
$$

For single-field models characterized by a slowly varying potential the spectral slope $n_{T}$ is given by 30]

$$
n_{T}=-\frac{M_{\mathrm{pl}}^{2}}{8 \pi}\left(\frac{V_{*}^{\prime}}{V_{*}}\right)^{2}
$$

where $V_{*}$ is the value of the inflationary potential when the scale associated to the present size of the horizon (corresponding to a frequency $f_{0}=(1 / 2 \pi) H_{0}$ ) crossed the horizon during the inflationary phase and $V_{*}^{\prime}$ is the first derivative of the inflaton potential at that point.Taking into account the frequency dependence of the spectral slope $(35)$, for frequencies $f \gg\left(H_{0} / 2 \pi\right)\left(1+z_{\text {eq }}\right)^{1 / 2}$ the spectrum reads 30 .

$$
\Omega_{\mathrm{GW}}=\frac{5}{2}\left(\frac{M_{*}}{M_{\mathrm{pl}}}\right)^{4}\left(\frac{\Omega_{r}}{1-\Omega_{\mathrm{de}}}\right)\left(\frac{f}{f_{0}}\right)^{n_{\mathrm{GW}}},
$$

where $M_{*}=V_{*}^{1 / 4}$ and

$$
n_{\mathrm{GW}}=n_{T}\left\{1-\frac{1}{2}\left[\left(n_{S}-1\right)-n_{T}\right] \log \frac{f}{f_{0}}\right\},
$$

where $n_{S}$ is the spectral index for scalar perturbations. A detailed analysis [16] using solutions of inflationary flow equations shows that for single-field slowroll inflationary models the maximum of the spectrum (36) compatible with WMAP data is $h_{0}^{2} \Omega_{\mathrm{GW}} \sim 5 \times 10^{-16}$ for frequencies $f \sim 100 \mathrm{~Hz}$ (see also [14, 17]).

\section{Gravitational-wave Background in Pre-big-bang Inflation}

In slow-roll inflation, the horizon and flatness problems are solved by postulating the presence of an epoch during which the energy-momentum tensor is dominated by the potential energy of a scalar field. This potential energy drives the phase of accelerated expansion, during which the field slowly rolls towards the minimum of the potential. In the 1990s, several attempts of building such cosmological setup in string theory encountered a number of problems 31$]^{2}$. Due to the presence of other fields, superstring theory at low energy

\footnotetext{
${ }^{2}$ For more recent successful attempts to build slow-roll inflationary models within string theory see, e.g., 32 .
} 
does not give Einstein general relativity - e.g., heterotic string theory in four dimensions is described by the action

$$
\Gamma_{\text {eff }}=\frac{1}{2 \lambda_{s}^{2}} \int d^{4} x \sqrt{|g|} e^{-\varphi}\left[\mathcal{R}+g^{\mu \nu} \partial_{\mu} \varphi \partial_{\nu} \varphi-\frac{1}{12}(d B)^{2}-V(\varphi)\right],
$$

where $\varphi$ is the dilaton field, related to the string coupling by $g^{2}=e^{\varphi}$; $d B=\partial_{\mu} B_{\nu \rho}+\partial_{\nu} B_{\rho \mu}+\partial_{\rho} B_{\mu \nu}$, where $B_{\mu \nu}$ is the two-form gauge field or antisymmetric field; $V(\phi)$ is a non-perturbative potential; and where $\lambda_{s}$ is the string scale. In writing (38) we disregard for simplicity the internal dimensions, whose dynamics can be described in terms of moduli fields [22]. Henceforth, we limit the discussion to the homogeneous and isotropic case with $B=0$ and $V=0\left[d s^{2}=-d t^{2}+a^{2}(t) d \boldsymbol{x}^{2}, \varphi=\varphi(t)\right]$.

In 1991 Veneziano [20] discovered that the solution of the low-energy string-effective action (38) satisfies the scale factor duality symmetry: $a(t) \rightarrow$ $1 / a(t), \varphi(t) \rightarrow \varphi(t)-6 \log a(t) 3$ with $a(t) \sim t^{1 / \sqrt{3}}$ and $\varphi(t) \sim-\log t$. Noticing this property, Veneziano [20] conceived the idea of implementing the inflationary phase at times before the would-be big-bang singularity, proposing the prebig-bang scenario. Indeed, it can be easily shown that for $t<0, \dot{a}>0, \ddot{a}>0$, thus the Universe undergoes a (super) inflationary phase. Two different but physically equivalent descriptions of the PBB phase exist: either the stringframe picture described by (38), where the Universe undergoes an accelerated expansion $(H>0, \dot{H}>0, \dot{\varphi}>0)$, or the Einstein-frame picture, where the action (38) has the standard Hilbert-Einstein form and the evolution of the Universe is described by an accelerated contraction, or gravitational collapse $(H<0, \dot{H}<0, \dot{\varphi}>0)$.

This new kind of inflation, which can be shown to solve the homogeneity and flatness conundra, is driven by the kinetic energy of the dilaton field and forces both the string coupling $(\dot{g}>0)$ and the spacetime curvature to grow toward the future (i.e., toward the stringy phase). As a consequence, at least in the homogeneous case, the inflationary stage lasts for ever $(t \rightarrow-\infty)$ and the initial state of the Universe is nearly flat, cold, and decoupled: $g \ll 1$, $\mathcal{R} \lambda_{s}^{2} \ll 1$.

The scale factor duality symmetry has constituted the basis of a class of inflationary models subsequently investigated by Gasperini and Veneziano [21, and it is also at the basis of the so-called ekpyrotic cosmological scenario 33. 34.

As far as metric perturbations are concerned, the most striking feature of the PBB spectra is a strong tilt toward high frequencies [36]. In [23, Veneziano and collaborators estimated the SGWB, obtaining

$$
\Omega_{\mathrm{GW}} \sim \frac{1}{z_{\mathrm{eq}}} g_{s}^{2}\left(\frac{f}{f_{s}}\right)^{3}\left\{\left[1+\frac{1}{2} \log \frac{f_{s}}{f}\right]+\frac{1}{z_{s}^{3}}\left(\frac{g_{1}}{g_{s}}\right)^{2}\right\}, \quad\left(f<f_{s},\right)
$$

\footnotetext{
${ }^{3}$ Here for convenience we fix the origin of time at $t=0$.
} 


$$
\Omega_{\mathrm{GW}} \sim \frac{g_{1}^{2}}{z_{\mathrm{eq}}}\left[\left(\frac{f}{f_{1}}\right)^{6-2 \beta}+\left(\frac{f}{f_{1}}\right)^{2 \beta}\right], \quad\left(f_{s}<f<f_{1}\right),
$$

where $g_{s}$ is the value of the string coupling at the onset of the high-curvature stringy phase, $f_{s}$ is the frequency corresponding to the lowest scale exiting during the dilaton-driven phase, $f_{1} \sim 10^{11} \mathrm{~Hz}$ is the ultraviolet cutoff, $g_{1}=$ $M_{s} / M_{\mathrm{pl}}, z_{s}$ is the redshift of the stringy phase, $\beta=-\log \left(g_{s} / g_{1}\right) / \log z_{s}$, and $z_{\text {eq }}$ is the redshift of matter-radiation equality.

\subsection{Pre-big-bang Minimal Model}

Here, we derive more in detail the SGWB following [35]. In the string frame, where strings follow geodesic trajectories, it is straightforward to show that the canonical variable $\Psi_{\mu \nu}$ associated to tensor perturbations is related to the metric by

$$
g_{\mu \nu}=a^{2}\left(\eta_{\mu \nu}+h_{\mu \nu}\right)=a^{2}\left(\eta_{\mu \nu}+\frac{g}{a} \Psi_{\mu \nu}\right)
$$

The Fourier modes of the two physical traceless and transverse polarization states satisfy the following wave equation:

$$
\Psi_{k}^{\prime \prime}+\left(k^{2}-V\right) \Psi_{k}=0
$$

where prime denotes differentiation with respect to the conformal time and $V=(g / a)^{\prime \prime} /(g / a)$. In the following, we shall restrict our attention to a class of minimal PBB models characterized by an initial accelerated, dilaton-driven phase followed by a stringy phase (during which $H$ and $d \varphi / d t$ are assumed to be approximately constant [37]) eventually evolving towards a standard radiation-dominated phase. During the dilaton-dominated regime $(-\infty<\eta<$ $\left.\eta_{s}<0\right)$ the scale factor and the dilaton field read

$$
\begin{gathered}
a(\eta)=-\frac{1}{H_{s} \eta_{s}}\left(\frac{\eta-(1-\alpha) \eta_{s}}{\alpha \eta_{s}}\right)^{-\alpha}, \\
\varphi(\eta)=\varphi_{s}-\gamma \log \frac{\eta-(1-\alpha) \eta_{s}}{\alpha \eta_{s}},
\end{gathered}
$$

where $\alpha=1 /(1+\sqrt{3}), \gamma=\sqrt{3}$. During the stringy phase $\left(\eta_{s}<\eta<\eta_{1}\right)$ one expects that higher-order terms saturate the growth of the curvature [37. Hence during this phase the scale factor and the dilaton can be parametrized as follows:

$$
\begin{gathered}
a(\eta)=-\frac{1}{H_{s} \eta}, \\
\varphi(\eta)=\varphi_{s}-2 \beta \ln \frac{\eta}{\eta_{s}} .
\end{gathered}
$$

Finally, assuming that a non-perturbative dilaton potential sets in stabilizing the dilaton, the radiation phase $\left(\eta_{1}<\eta<\eta_{r}\right)$ is described by 


$$
a(\eta)=\frac{1}{H_{s} \eta_{1}^{2}}\left(\eta-2 \eta_{1}\right) .
$$

For those three different phases the potential $V$ reads

$$
\begin{gathered}
V(\eta)=\frac{1}{4}\left(4 \nu^{4}-1\right)\left[\eta-(1-\alpha) \eta_{s}\right]^{-2}, \quad-\infty<\eta<\eta_{s}, \\
V(\eta)=\frac{1}{4}\left(4 \mu^{4}-1\right) \eta^{-2}, \quad \eta_{s}<\eta<\eta_{1}, \\
V(\eta)=0, \quad \eta_{1}<\eta<\eta_{r} .
\end{gathered}
$$

where, $2 \nu=|2 \alpha-\gamma+1|, 2 \mu=|2 \beta-3|$. The exact solutions of (42) in the three phases are

$$
\begin{gathered}
\Psi_{k}=\sqrt{\left|\eta-\left(1-\alpha \eta_{s}\right)\right|} H_{\nu}^{(2)}\left(k\left|\eta-\alpha \eta_{s}\right|\right), \quad-\infty<\eta<\eta_{s}, \\
\Psi_{k}=\sqrt{|\eta|}\left[A_{+} H_{\mu}^{(2)}(k|\eta|)+A_{-} H_{\mu}^{(1)}(k|\eta|)\right], \quad \eta_{s}<\eta<\eta_{1}, \\
\Psi_{k}=i \sqrt{\frac{2}{\pi k}}\left[B_{+} e^{-i k \eta}-B_{-} e^{i k \eta}\right], \quad \eta_{1}<\eta<\eta_{r},
\end{gathered}
$$

where $H_{\mu, \nu}^{(1,2)}$ are Hankel's functions of the first and second kind. The Bogoliubov coefficients $A_{ \pm}, B_{ \pm}$can be computed by requiring the continuity of the Fourier modes and its first derivative on the space-like surfaces $\eta=\eta_{s}$ and $\eta=\eta_{1}$. The result for the spectrum is

$$
\begin{gathered}
\Omega_{\mathrm{GW}}(f)=a(\mu) \frac{\left(2 \pi f_{s}\right)^{4}}{H_{0}^{2} M_{\mathrm{pl}}^{2}}\left(\frac{f_{1}}{f_{s}}\right)^{2 \mu+1}\left(\frac{f}{f_{s}}\right)^{5-2 \mu} \mid H_{\nu}^{(2)^{\prime}}\left(\frac{\alpha f}{f_{s}}\right) J_{\mu}\left(\frac{f}{f_{s}}\right) \\
-H_{\nu}^{(2)}\left(\frac{\alpha f}{f_{s}}\right) J_{\mu}^{\prime}\left(\frac{f}{f_{s}}\right)+\left.\frac{(1-\alpha)}{2 \alpha} \frac{f_{s}}{f} H_{\nu}^{(2)}\left(\frac{\alpha f}{f_{s}}\right) J_{\mu}\left(\frac{f}{f_{s}}\right)\right|^{2}
\end{gathered}
$$

where

$$
a(\mu)=\frac{\alpha}{48} 2^{2 \mu}(2 \mu-1)^{2} \Gamma^{2}(\mu) .
$$

For the class of cosmological models under consideration $\nu=0$; hence, using the identity $H_{0}^{(2)^{\prime}}(z)=-H_{1}^{(2)}(z)$, the spectrum is given by

$$
\begin{aligned}
& \Omega_{\mathrm{GW}}(f)=a(\mu) \frac{\left(2 \pi f_{s}\right)^{4}}{H_{0}^{2} M_{\mathrm{pl}}^{2}}\left(\frac{f_{1}}{f_{s}}\right)^{2 \mu+1}\left(\frac{f}{f_{s}}\right)^{5-2 \mu} \mid H_{0}^{(2)}\left(\frac{\alpha f}{f_{s}}\right) J_{\mu}^{\prime}\left(\frac{f}{f_{s}}\right) \\
& +H_{1}^{(2)}\left(\frac{\alpha f}{f_{s}}\right) J_{\mu}\left(\frac{f}{f_{s}}\right)-\left.\frac{(1-\alpha)}{2 \alpha} \frac{f_{s}}{f} H_{0}^{(2)}\left(\frac{\alpha f}{f_{s}}\right) J_{\mu}\left(\frac{f}{f_{s}}\right)\right|^{2} .
\end{aligned}
$$

Assuming that the curvature scale at the onset of the string scale is $H_{s} \sim$ $1 / \lambda_{s} \sim g_{\text {gut }} M_{\mathrm{pl}} \sim 0.015 M_{\mathrm{Pl}}$ and that the cosmic time value at which the 
stringy phase ends is $t_{1} \sim \lambda_{s}$ the peak frequency is $f_{1} \sim 4.3 \times 10^{10} \mathrm{~Hz}$. Hence the spectrum depends on two arbitrary parameters, $f_{s}$ and $\beta$. (Note that (3) can be recovered with the following mapping: $z_{s}=f_{1} / f_{s}$ and $g_{s} / g_{1}=$ $\left(f_{s} / f_{1}\right)^{\beta}$, with $\beta$ given by $2 \mu=|2 \beta-3|$.)

From (55), one finds that the maximum value of the spectrum compatible with the BBN and CMB bounds is

$$
h_{0}^{2} \Omega_{\mathrm{GW}}^{\max } \sim 3.0 \times 10^{-7} .
$$

Such value is quite interesting, since it is about one order of magnitude below the sensitivity of first-generation LIGO interferometers, and well above the sensitivity of second-generation interferometers, such as advanced LIGO.

\subsection{Pre-big-bang Non-minimal Models}

The SGWB in the minimal PBB model was originally evaluated neglecting the higher-curvature corrections in the equation of tensorial fluctuations during the stringy phase. Gasperini 38] evaluated the higher-order equation for tensorial fluctuations and showed that these corrections modify the amplitude of the perturbation only by a factor of order 1 .

In [39, 40] the authors examined the effect of radiation produced during reheating processes occurring below the string scale. Such processes may be needed in the PBB model to dilute relic particles produced during (or at the very end of) the $\mathrm{PBB}$ phase. The abundance of those particles could spoil the BBN predictions 41. Depending on when and for how long the entropy is produced, it can change the shape and reduce the amplitude of the SGWB. If we assume that the reheating process occurs at the end of the stringy phase (i.e., all the entropy is produced at the end of the stringy phase), then the effect of the process is a simple scaling of the original spectrum by the factor $(1-\delta s)^{4 / 3}$, where $\delta s$ is the fraction of the present thermal entropy density that the reheating process produced.

Finally, as first noticed in [39, it is well possible that many more cosmological phases are present between the pre- and the post-big-bang cosmological phases (see, e.g., [39, 42 ). If this is the case, the GW spectra during the high-curvature and/or strong coupling region will be characterized by several branches with increasing and decreasing slopes. Due to the dependence of the spectra on a larger number of parameters, it would be more difficult to constrain these non-minimal scenarios using GW detectors.

\section{Accessibility of LIGO to Pre-big-bang Models}

We shall now discuss the implications of recent and future analysis of LIGO on PBB models, notably on its parameter space. 


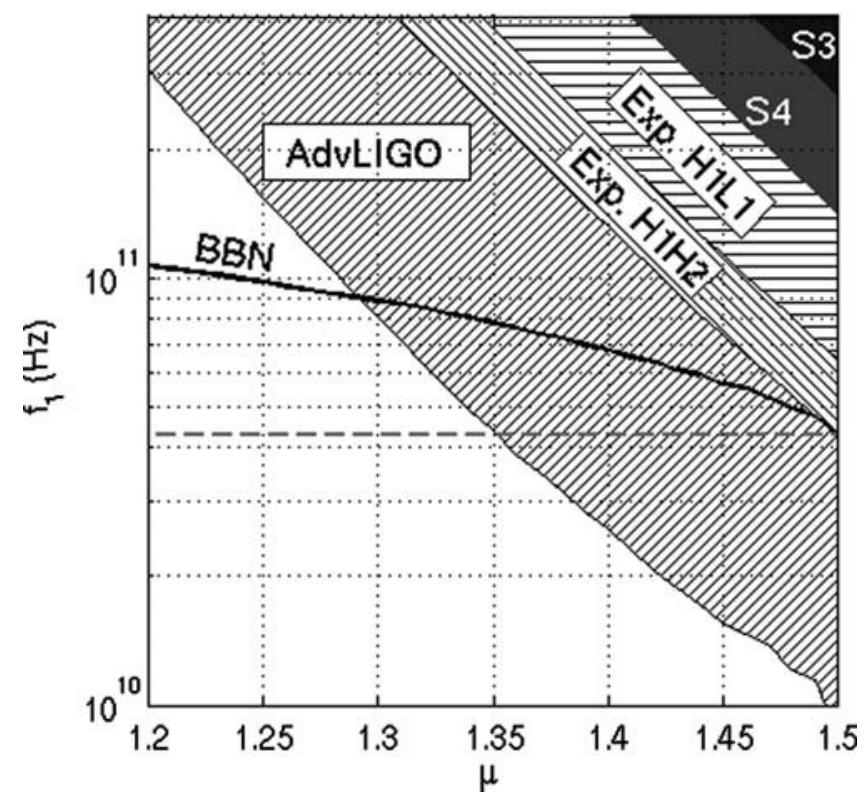

Fig. 1. The $f_{1}-\mu$ plane with $f_{s}=30 \mathrm{~Hz}$ [18. The shaded regions are excluded by the LIGO S3 upper limit (darker) and by the LIGO S4 limit. The hatched regions are accessible to future LIGO runs, assuming an observation time of 1 year: the predicted sensitivity for the H1L1 pair, assuming first design configuration (-); expected LIGO sensitivity for the $\mathrm{H} 1 \mathrm{H} 2$ pair, assuming first design configuration ( $\backslash$ ); expected Advanced LIGO sensitivity for the H1H2 pair, assuming interferometer configuration optimized for the binary neutron star inspiral search (/). The solid black curve is the exclusion curve consistent with the nucleosynthesis limit (the excluded region is above the curve). The horizontal dashed line denotes the value of $f_{1}=4.3 \times 10^{10} \mathrm{~Hz}$ (courtesy of LIGO)

As already pointed out in the previous section, in minimal PBB models the spectrum (55) is characterized by the following parameters: (i) the dimensionless quantity $\mu=|2 \beta-3|$ which is positive definite and constrained to be $\mu \leq 3 / 2$, since for $\mu>3 / 2$ the spectrum would be incompatible with the existing experimental bounds and (ii) the frequency parameter $f_{s}$ which is defined so that $0<f_{s}<f_{1}$. Since the spectrum (55) behaves as $\left(f / f_{s}\right)^{3}$ for $f<f_{s}$, a comparison with LIGO data is insensitive to values of $f_{s}$ above the interferometer band. In particular, one finds that LIGO data can scan values $f_{s}<30 \mathrm{~Hz}[18$. Furthermore, in the high-frequency limit, the value of the spectrum is independent on $f_{s}$. The other parameter is the frequency $f_{1}$ (which defines the peak frequency of the spectrum) 4

\footnotetext{
${ }^{4}$ In the more common version of the minimal PBB model [22, 23, 42, the frequency $f_{1}$ is obtained by imposing that the energy density becomes critical at the beginning of the radiation phase and that the photons we observe
} 


$$
f_{1} \sim 4.3 \times 10^{10} \mathrm{~Hz}\left(\frac{H_{s}}{0.15 M_{\mathrm{pl}}}\right)\left(\frac{t_{1}}{\lambda_{s}}\right)^{1 / 2}
$$

where $H_{s}$ is the curvature scale at the onset of the intermediate stringy phase and $t_{1}$ is the value of the cosmic time at which such phase ends. Even assuming $H_{s} \sim \lambda_{s}^{-1}, t_{1} \sim \lambda_{s}$ since the spectrum (55) has a strong dependence on $f_{1}$ $\left(\Omega_{\mathrm{GW}} \propto f_{1}^{4}\right)$, an order of magnitude combined variation in $t_{1}, H_{s}$ can yield quite a large variation in the spectrum.

Based on a previous analysis carried out in [19, during the last scientific run, the LIGO scientific collaboration has scanned the three-dimensional parameter space $\mu, f_{s}, f_{1}$ using $192 \mathrm{~s}$ - long intervals with $1 / 32 \mathrm{~Hz}$ resolutionassessing the accessibility of LIGO to each of the PBB parameters describing the spectrum (18). Furthermore, the design sensitivity of the initial and advanced LIGO configuration was taken into account. A summary of the results is shown in Fig. 1, where the $f_{1}-\mu$ plane is considered (fixing $f_{s}=30 \mathrm{~Hz}$ ). The results pertaining the third (S3) and fourth (S4) LIGO scientific runs provide a first, albeit quite restricted, scanning of the parameter space. The indirect BBN bound is still quite a strong constraint, but future and longer runs of the LIGO interferometers are expected to enlarge the available part of the parameter space, eventually overcoming the BBN bound.

\section{Conclusions}

The most interesting and robust feature of the relic background of gravitational radiation predicted by the $\mathrm{PBB}$ model is the positive spectral slope $\left(n_{T}=3\right)$ at low frequency, i.e., for modes that exit the horizon during the dilaton-driven (super) inflationary phase and re-enter during radiationdominated era. Such attribute is a consequence of the Universe's equation of state during the (super) inflationary PBB phase and is shared by other nonconventional cosmological models. For example in quintessential inflationary model [43, where the standard radiation-dominated era is preceded by a phase characterized by a stiffer equation of state, the SGWB increases linearly with frequency. A blue primordial spectrum of GWs could be produced in a class of models with superluminal $(w=p / \rho<1)$ equation of state 44, as discussed in [45], where the inflaton field is characterized by a non-local Lagrangian. Furthermore, in other cosmological setups based upon superstring theory, as the the cyclic/ekpyrotic models [46, the GW spectrum increases as function of frequency, although its amplitude normalization makes it unobservable by ground- and space-based detectors.

today originated from the amplified vacuum fluctuations during the dilatondriven inflationary phase. Within these assumptions (57) can be re-written as $f_{1} \simeq g_{1}^{1 / 2}\left(H_{s} /\left(0.15 M_{\mathrm{Pl}}\right)\right)^{1 / 2}\left(H_{0} M_{\mathrm{Pl}}\right)^{1 / 2} \Omega_{\gamma}^{1 / 4}$, where $\Omega_{\gamma}=4 \times 10^{-5} h_{0}^{-2}$ and $g_{1}$ is the string coupling at the end of the stringy phase. 
The blue spectrum predicted by PBB models yields to a lack of tensorial contributions to the CMB temperature and polarization anisotropies. Therefore, the detection of a tensorial component of primordial origin in the CMB fluctuations would rule out the current version of the PBB model.

Finally, it is worth to notice that during the initial dilaton-driven inflationary phase characteristic of $\mathrm{PBB}$ models the initial (classical) tensor inhomogeneities are not de-amplified [47, as it occurs in slow-roll inflation. This result, though paradoxical, does not imply that the initial value of tensor inhomogeneities must be fine tuned to an unnaturally small value. Indeed, it can be shown [47] that the energy density of such tensor classical fluctuations is indeed de-amplified. However, in order to solve the homogeneity problem in those superstring-inspired models, more tighter constraints [4] than in slow-roll inflation models ought to be imposed.

All that said, future ground- and space-based detectors will be in the unique and privileged position of either detect the PBB SGWB or put relevant bounds on the parameter space of the string-cosmology scenario originally proposed by Gabriele Veneziano.

\section{References}

1. http://www . ligo.org 845

2. http://www.virgo.pi.infn.it 845

3. http://www.geo600.uni-hannover.de 845

4. http://www.tama.mtk.nao.ac.jp 845

5. http://lisa.jpl.nasa.gov 845

6. http://science.hq.nasa.gov/universe/science/bang.html 845

7. N. Seto, S. Kawamura, T. Nakamura: Phys. Rev. Lett. 87, 221103 (2001) 845

8. E. Iacopini, E. Picasso, F. Pegoraro, L. A. Radicati: Phys. Lett. A 73, 140 (1979); C.M. Caves: Phys. Lett. B 80, 323 (1979) 845

9. A.M. Cruise: Class. Quant. Grav. 17, 2525 (2000); A.M. Cruise, R. Ingley: Class. Quant. Grav. 22, S497 (2004) 846

10. http://www.skatelescope.org 846

11. B. Allen, "The stochastic gravity-wave background: sources and detection," [gr-qc/9604033] 846, 849]

12. M. Maggiore Phys. Rep. 331, 283 (2000) 846

13. L.P. Grishchuk: Sov. Phys. JEPT 40, 409 (1975); A.A. Starobinski: JEPT Lett. 30, 682 (1979); V.A. Rubakov, M. Sazhin, A. Veryaskin: Phys. Lett. B 115, 189 (1982); R. Fabbri, M. Pollock: Phys. Lett. B 125, 445 (1983); L.F. Abbott, D.D. Harari: Nucl. Phys. B 264, 487 (1986); B. Allen: Phys. Rev. D 37, 2078 (1988); V. Sahni: Phys. Rev. D 42, 453 (1990) 846847

14. L. A. Boyle, P. J. Steinhardt, N. Turok: Phys. Rev. Lett. 96, 311101 (2006) 846 853

15. L. A. Boyle, P. J. Steinhardt: "Probing the early universe with inflationary gravitational waves," [astro-ph/0512014] 846 847]

16. B. C. Friedman, A. Cooray, A. Melchiorri: "WMAP-normalized inflationary model predictions and the search for primordial gravitational waves with direct detection experiments," [astro-ph/0610220]. 846] 853 
17. T.L. Smith, M. Kamionkowski, A. Cooray: Phys. Rev. D 73023504 (2006) 847 853

18. B. Abbott et al.: "Searching for a stochastic background of gravitational waves with LIGO," [astro-ph/0608606] 847 858

19. V. Mandic, A. Buonanno: Phys. Rev. D 73, 063008 (2006) 847859

20. G. Veneziano: Phys. Lett. B 265, 287 (1991) 847,854

21. M. Gasperini, G. Veneziano: Astropart. Phys. 1, 317 (1993); Mod. Phys. Lett. A 8, 3701 (1993); Phys. Rev. D 50, 251 (1994) 847 854

22. M. Gasperini, G. Veneziano: Phys. Rep. 373, 1 (2003) 847, 854, 858

23. R. Brustein, M. Gasperini, M. Giovannini, G. Veneziano, Phys. Lett. B 361, 45 (1995) 847854858

24. C.J. Copi, D.N. Schramm, M.S. Turner: Phys. Rev. D 55, 3389 (1997). 847

25. T. Smith, E. Pierpaoli, M. Kamionkowski: Phys. Rev. Lett. 97, 021301 (2006) 847

26. G. Veneziano: "String cosmology: concepts and consequences," [hepth/9512091] 848

27. B. Allen: Phys. Rev. D 37, 2078 (1988) 851

28. H. Kodama, M. Sasaki: Suppl. Prog. Theor. Phys. 78, 1 (1984); V. F. Mukhanov, H. A. Feldman, R. H. Brandenberger: Phys. Rep. 215, 203 (1992) 850

29. W. H. Kinney, E. W. Kolb, A. Melchiorri, A. Riotto: Phys. Rev. D 74, 023502 (2006) 852

30. M. Turner: Phys. Rev. D 55, 435 (1997) 853

31. B.A. Campbell, A.D. Linde, K.A. Olive: Nucl. Phys. B 335, 146 (1991); R. Brustein, P.J. Steinhardt: Phys. Lett. B 302, 196 (1993) 853

32. S. Kachru, R. Kallosh, A. Linde, J. Maldacena, L. McAllister, S.P. Trivedi: JHEP 0408, 030 (2004) 853

33. J. Khoury, B.A. Ovrut, P.J. Steinhardt, N. Turok: Phys. Rev. D 64, 123522 (2001) 854

34. J. Khoury, B.A. Ovrut, N. Seiberg, P.J. Steinhardt and N. Turok: Phys. Rev. D 65, 086007 (2002) 854

35. A. Buonanno, M. Maggiore, C. Ungarelli: Phys. Rev. D 55, 3330 (1997) 855

36. R. Brustein, M. Gasperini, M. Giovannini, V. F. Mukhanov, G. Veneziano, Phys. Rev. D 51, 6744 (1995) 854

37. M. Gasperini, M. Maggiore, G. Veneziano: Nucl. Phys. B 494, 315 (1996). 855

38. M. Gasperini: Phys. Rev. D 56, 4815 (1997). 857

39. M. Gasperini, "Relic gravitons from the pre-big bang: what we know and what we do not know," [hep-th/9607146] 857

40. R. Brustein, M. Gasperini, G. Veneziano: Phys. Rev. D 55, 3882 (1997) 857

41. A. Buonanno, M. Lemoine, K.A. Olive: Phys. Rev. D 62, 083513 (2000) 857

42. A. Buonanno, K. Meissner, C. Ungarelli, G. Veneziano: JHEP 001, 004 (1998) 857858

43. P.J.E. Peebles, A. Vilenkin: Phys. Rev. D 59, 063505 (1999); M. Giovannini: Phys. Rev. D 60, 123511 (1999) 859

44. L. Grishchuk: "Relic gravitational waves and cosmology", [astro-ph/0504018] 859]

45. M. Baldi, F. Finelli, S. Matarrese: Phys. Rev. D 72, 083504 (2005) 859

46. L. A. Boyle, P. Steinhardt, N. Turok: Phys. Rev. D 69, 127302 (2004) 859

47. A. Buonanno, T. Damour: Phys. Rev. D 50, 3713 (2001) 860 